\title{
Current Perspectives on Transport Medicine in Pediatric Hospital Medicine Fellowships
}

\author{
Mark H. Corden, MD ${ }^{1,2 *}$, Thanh Huynh, MD ${ }^{1,2}$, Purnima Mandal, MD ${ }^{1,2}$, Sanjay Chand ${ }^{1}$, Jennifer Maniscalco, MD, MPH ${ }^{1,2}$
}

'Division of Hospital Medicine, Department of Pediatrics, Children's Hospital Los Angeles, Los Angeles, California; ${ }^{2}$ Department of Pediatrics, Keck School of Medicine, University of Southern California, Los Angeles, California.

Transport medicine (TM) is a Pediatric Hospital Medicine (PHM) Core Competency and part of the proposed PHM fellowship curricular framework. No published TM curricula are available. This cross-sectional study was designed to determine attitudes toward TM training among PHM fellowship stakeholders and conduct a TM curriculum needs assessment. Unique, web-based, anonymous surveys for PHM fellows, graduates, and program directors (PDs) were administered, with response rates of $57 \%, 37 \%$, and $44 \%$, respectively. Fellows' interest in completing a TM rotation is greater than their perceived interest by PDs $(P=.06)$. Graduates who completed a TM rotation were more likely to recommend a TM rotation than those who did not $(P=$ .001). Perceived barriers included lack of a formal TM curriculum and time constraints. Stabilizing patients and triage of referrals were deemed important learning objectives, and active learning strategies were prioritized. Curriculum design should focus on topics specific to the transport process and environment. Journal of Hospital Medicine 2018;13:770-773. Published online first April 25,2018 . (C) 2018 Society of Hospital Medicine
T ransport medicine (TM) involves the provision of care to patients who require transfer to a healthcare facility that can deliver definitive treatment. ${ }^{1}$ Pediatric interfacility transport occurs in approximately $10 \%$ of nonneonatal, nonpregnancy pediatric hospitalizations in the United States. ${ }^{2}$ Studies document a decline in resident participation in pediatric transports and variability in curricular content. ${ }^{3,4}$ As a result, pediatric hospitalists, who often serve as the referring, accepting, transport, and/or medical control physician during interfacility transports, 5,6 may have gaps in training related to TM.

The Pediatric Hospital Medicine (PHM) Core Competencies include "Transport of the Critically III Child." Additionally, the Curriculum Committee of the PHM Fellowship Directors Council proposed a curricular framework that includes a required clinical experience in "Care and Stabilization of the Critically III Child, "8 which can occur in a variety of practice settings, including TM. TM is also listed as a potential elective rotation.

In 2014, 60\% of PHM fellowships included a required or optional TM rotation. ${ }^{9}$ A recent study of pediatric emergency, critical care, and neonatal medicine fellowships revealed a paucity of formal or published TM curricula in these programs. ${ }^{10}$ Furthermore, no standard or published TM curricula have been

\footnotetext{
*Address for correspondence: Mark Corden, MD, Division of Hospital Medicine, Department of Pediatrics, Children's Hospital Los Angeles, 4650 Sunset Blvd, MS 94, Los Angeles, CA 90027; Telephone: (323) 361-6177; Fax: (323) 361 8106; E-mail: mcorden@chla.usc.edu
}

Received: August 15, 2017; Revised: January 20, 2018;

Accepted: February 1, 2018

Society of Hospital Medicine DOI 10.12788/jhm.2962 established for PHM fellowships. The primary objective of our study is to determine attitudes regarding TM training among PHM fellows, recent PHM fellowship graduates, and PHM fellowship program directors (PDs). The secondary objective is to identify how the perspectives of these fellowship stakeholders could influence the design of a TM curriculum.

\section{METHODS}

This cross-sectional study focused on three stakeholder groups related to PHM fellowships. The subjects included in the study were physicians enrolled in a PHM fellowship (fellow) during the 2015-2016 academic year, graduates of fellowship (graduate) between 2010 and 2015, and fellowship program directors (PD). Unique web-based, anonymous surveys for each group were developed, reviewed by content and methodology experts, and piloted with local pediatric hospitalists. Surveys consisted of unfolding multiple-choice questions and ranking items along Likert scales and the Dreyfus model.

Questions were designed to elicit demographic data, perspectives, and experience related to TM education in PHM fellowships across all respondent groups. Depending on the context, identical or similar questions were asked among the groups. For example, all groups were asked to prioritize learning objectives for a TM rotation. Graduates and PDs reported the most effective teaching methods for use during a TM rotation. Fellows rated their own interest in a TM elective, and PDs were asked to rate the level of interest among their fellows.

Participant contact information was obtained from a website (phmfellows.org) and databases of fellows and graduates, which are maintained by the PHM Fellowship Directors Council (personal communication, Jayne Truckenbrod, DO; February 2, 2017). Between February and April 2016, the participants were 
TABLE. Perceived Barriers and Learning Objectives for a Transport Medicine Rotation

\begin{tabular}{|c|c|c|c|}
\hline Rank & Fellows $^{a}$ & Graduates & Program Directors \\
\hline \multicolumn{4}{|c|}{ BARRIERS } \\
\hline 1 & Lack of relevance to a career in hospital medicine & Lack of formal transport medicine curriculum & Lack of formal transport medicine curriculum \\
\hline 2 & No reservations & Transport rotation not offered at my fellowship program & $\begin{array}{l}\text { Transport medicine is not as relevant as other core competencies } \\
\text { in Pediatric Hospital Medicine }\end{array}$ \\
\hline 3 & $\begin{array}{l}\text { Lack of time during fellowship for participating in a } \\
\text { transport medicine rotation }\end{array}$ & Transport service not structured to accommodate trainees & $\begin{array}{l}\text { Lack of time during fellowship to allow trainee to participate in a } \\
\text { transport rotation }\end{array}$ \\
\hline \multicolumn{4}{|c|}{ LEARNING OBJECTIVES } \\
\hline 1 & Appropriate triage of referrals & Stabilizing patients for transport & Stabilizing patients for transport \\
\hline 2 & $\begin{array}{l}\text { Learning to work within the constraints of the resources } \\
\text { available }\end{array}$ & Appropriate triage of referrals & Appropriate triage of referrals \\
\hline 3 & Stabilizing patients for transport & $\begin{array}{l}\text { Understanding the different types of medical transport AND } \\
\text { Management of critically ill children }\end{array}$ & Management of critically ill children \\
\hline
\end{tabular}

${ }^{a}$ Fellows were asked to identify reservations (rather than barriers) to participating in a Transport Medicine rotation.

individually emailed a link to their respective surveys, and three reminder e-mails were sent to nonresponders. The survey was administered through SurveyMonkey (www.surveymonkey.com).

SPSS (IBM SPSS Statistics, IBM Corporation, Armonk, New York) was used for statistical analysis. Descriptive data were presented using mean and standard deviation. Comparisons among fellows, graduates, and PDs were conducted using one-way analyses of variance or Mann-Whitney $U$ test. Frequency of application and self-evaluation of core competency skills before and after the rotation were evaluated using paired sample $t$-tests. The study protocol was deemed exempt from review by our local Institutional Review Board.

\section{RESULTS}

Forty of $70(57 \%)$ fellows, 32 of 87 graduates (37\%), and 14 of 32 PDs (44\%) responded to the survey. The majority of the participants described their respective programs as two years in duration (59\% for fellows, $56 \%$ for graduates, and $85 \%$ for PDs). Most programs (85\%) were based at children's hospitals. Most graduates (84\%) practiced in a children's hospital, and $12 \%$ of them practiced in a community site or a combination of sites.

Both fellows and graduates reported limited involvement in several aspects of TM prior to fellowship. Fellows' interest in completing a TM rotation during fellowship is greater than the interest as perceived by PDs $(3.03 \pm 1.00$ vs $2.38 \pm 1.19, P=$ .061). Prior TM exposure in residency or perceived proficiency in TM was not associated with lack of interest. Twenty-five percent of graduates completed a TM rotation during PHM fellowship. Many graduates agreed (41\%) or strongly agreed (16\%) with the statement "I recommend participating in a TM rotation during PHM fellowship." Graduates who had completed a TM rotation were more likely to agree with this statement $(P=.001)$.

There were similarities between reservations about participating in a TM rotation among fellows and barriers identified by graduates and PDs (Table). However, no graduates cited lack of relevance to a career in PHM as a barrier to participation in a TM rotation. Fellows, graduates, and PDs reported concordant responses regarding the prioritization of learning objectives for a TM rotation (Table). Both graduates and PDs ranked active learning strategies, such as direct patient care and simulation, as the most effective methods for teaching TM.

Discordance was noted between how frequently fellows participated in aspects of TM during fellowship and graduates' current practice of PHM (Figure). With regard to select TM-related PHM core competencies, such as respiratory failure, shock, and leading a healthcare team, most (63\%-90\%, depending on the competency) fellows perceived themselves as "competent" prior to the start of the fellowship. Nevertheless, more than $70 \%$ of fellows remained very or extremely interested in gaining additional experience in each competency during fellowship.

\section{DISCUSSION}

Survey respondents demonstrate variable levels of interest and engagement in TM training; in particular, fellows and graduates often reported greater interest and value in a TM rotation than PDs. Similar to fellows in related fields, ${ }^{10}$ PHM fellows and graduates selected clinical topics as the most essential elements of TM training. In accordance with the literature, our findings suggest that direct patient care, one-on-one instruction, and simulation would be appropriate and popular methods for delivering this type of educational content. ${ }^{10,11}$

Curriculum design for a TM rotation should reinforce clinical PHM competencies related to TM while focusing on topics that are specific to the transport environment, such as methods of interfacility transport, handoffs, transitions of care, and team leadership. 2,7,12 Trainee comfort level with different forms of transport (eg, fear of flying, motion sickness) and local and state policies regarding interfacility transfer should also be 


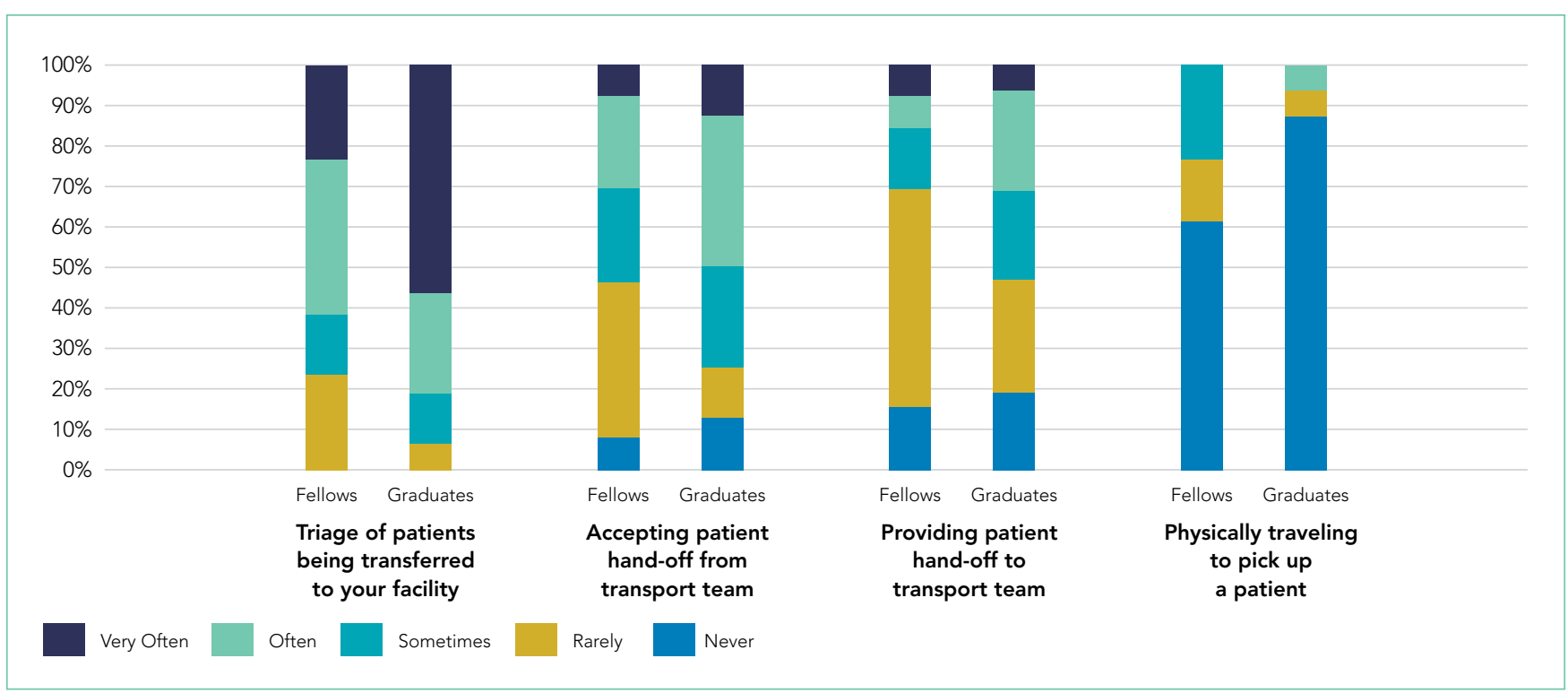

FIG. Frequency of participation in certain aspects of transport medicine, fellows (as reported by program directors) versus graduates.

considered. In addition, fellows could engage in clinical research and quality improvement projects related to TM given the overall paucity of literature in the field. ${ }^{13}$

Several reasons can explain why fellows and graduates place a greater value on a TM rotation than PDs. Fellows and graduates may perceive inherent value in gaining particular knowledge and skills, such as greater understanding of the logistics and personnel involved in transferring patients and experience working with a healthcare team in a unique and dynamic setting., ${ }^{3,10,14}$ Meanwhile, PDs may have had limited personal exposure to TM or may underestimate the limited exposure to TM for fellows while in residency. ${ }^{3}$

PDs may not be aware of the extent of participation in elements of transport among graduates. A recent workforce survey of pediatric interfacility transport systems indicated that although medical directors are from the fields of emergency, critical care, and neonatal medicine, $20 \%$ of medical control physicians are pediatric hospitalists. ${ }^{4}$ Given that the majority of PHM fellowships are based at children's hospitals and transport teams are often associated with intensive care or emergency medicine units, PDs may have limited exposure to transport systems that incorporate hospitalists.

Pediatric hospitalists at all practice sites must have clinical and systems skills related to TM. However, the scope of practice for those working at community sites may be more likely to include distinct elements of TM. ${ }^{6}$ Currently, most fellowship graduates work at free-standing children's or university-affiliated hospitals and have pursued careers in academic medicine. ${ }^{15}$ As the field evolves, the number of fellowship-trained pediatric hospitalists working at community sites may increase, making the acquisition of skills relevant to TM during fellowship training more crucial.

This study has several limitations. We attempted to identify all recent PHM fellowship graduates, but sampling bias may exist. Response bias may have been introduced by the self-re- porting of skill and proficiency as well as by the small sample size and response rate for some stakeholder groups. The latter may be exacerbated by the fact that we do not have data on the degree or distribution of program representation among the fellow and graduate groups, given the lack of identifying information collected. Finally, we did not collect specific information about existing TM curricula in PHM fellowships.

We report a variable level of interest and engagement in TM among fellowship stakeholders, even though "Transport of the Critically III Child" is a PHM Core Competency. Fellows are interested in TM but unsure of its relevance to a PHM career. Graduates support the acquisition of transport skills during fellowship training. We found agreement about the opportunity to teach core PHM knowledge and skills through a TM experience. Formal curricula, locally and nationally, could improve trainees' transport skills and provide a means for addressing an essential component of the proposed PHM fellowship curricular framework.

\section{Acknowledgments}

The authors would like to thank Tony Woodward, MD for reviewing the survey tools; Sheree Schrager, PhD and Margaret Trost, MD for their valuable insights into the results; and Grant Christman, MD for reviewing the manuscript.

Disclosures: The authors declare no potential conflicts of interest.

Funding: No funding was secured for this study.

\section{References}

1. Insoft RM, Schwartz HP, Romito J. Guidelines for Air and Ground Transport of Neonatal and Pediatric Patients., $4^{\text {th }}$ ed. Elk Grove Village, IL: American Academy of Pediatrics; 2016.

2. Rosenthal JL, Romano PS, Kokroko J, Gu W, Okumura MJ. Profiling interfacility transfers for hospitalized pediatric patients. Hosp Pediatr. 2017;7(6):335-343.

3. Kline-Krammes S, Wheeler DS, Schwartz HP, Forbes M, Bigham MT. Missed opportunities during pediatric residency training. Report of a 10-year follow-up survey in critical care transport medicine. Pediatr Emerg Care. 2012;28(1):1-5

4. Tanem J, Triscari D, Chan M, Meyer MT. Workforce survey of pediatric inter- 
facility transport systems in the United States. Pediatr Emer Care. 2016;32(6): 364-370.

5. Freed GL, Dunham KM. Pediatric hospitalists: training, current practice, and career goals. J Hosp Med. 2009;4(3):179-186.

6. Roberts KB. Pediatric hospitalists in community hospitals: hospital-based generalists with expanded roles. Hosp Pediatr. 2015;5(5):290-292.

7. Stucky ER, Maniscalco J, Ottolini MC, et al. The Pediatric Hospital Medicine Core Competencies Supplement: a Framework for Curriculum Development by the Society of Hospital Medicine with acknowledgement to pediatric hospitalists from the American Academy of Pediatrics and the Academic Pediatric Association. J Hosp Med. 2010;5(suppl 2):i-xv, 1-114.

8. Jerardi KE, Fisher E, Rassbach C, et al. Development of a Curricular Framework for Pediatric Hospital Medicine Fellowships. Pediatrics. 2017;140(1):1-8

9. Shah NH, Rhim HJH, Maniscalco J, Wilson K, Rassbach C. The current state of pediatric hospital medicine fellowships: A survey of program directors. $J$ Hosp Med. 2016;11(5):324-328.
10. Mickells GE, Goodman DM, Rozenfeld RA. Education of pediatric subspecialty fellows in transport medicine: a national survey. BMC Pediatrics. 2017;17(1):13.

11. Cross $B$, Wilson D. High-fidelity simulation for transport team training and competency evaluation. Newborn Inf Nurs Rev. 2009;9(4):200-206.

12. Weingart $C$, Herstich $T$, Baker $P$, et al. Making good better: implementing a standardized handoff in pediatric transport. Air Med J. 2013;32(1):40-46.

13. Kandil SB, Sanford HA, Northrup V, Bigham MT, Giuliano Jr. JS. Transport disposition using transport risk assessment in pediatrics (TRAP) score. Prehosp Emerg Care. 2012;16(3):366-373

14. Giardino AP, Tran XG, King J, Giardino ER, Woodward GA, Durbin DR. A longitudinal view of resident education in pediatric emergency interhospital transport. Pediatr Emerg Care. 2010;26(9):653-658.

15. Oshimurua JM, Bauer BD, Shah N, Nguyen N, Maniscalco J. Current roles and perceived needs of pediatric hospital medicine fellowship graduates. Hosp Pediatr. 2016;6(10):633-637 